\title{
THE REFOCUSING DISTANCE OF A STANDARD PLENOPTIC PHOTOGRAPH
}

\author{
Christopher Hahne, Amar Aggoun, Vladan Velisavljevic \\ christopher.hahne@study.beds.ac.uk; \{amar.aggoun; vladan.velisavljevic\}@beds.ac.uk \\ Dept. of Computer Science \& Technology, University of Bedfordshire \\ Park Square, Luton, Bedfordshire, LU1 3JU, United Kingdom
}

\begin{abstract}
In the past years, the plenoptic camera aroused an increasing interest in the field of computer vision. Its capability of capturing three-dimensional image data is achieved by an array of micro lenses placed in front of a traditional image sensor. The acquired light field data allows for the reconstruction of photographs focused at different depths. Given the plenoptic camera parameters, the metric distance of refocused objects may be retrieved with the aid of geometric ray tracing. Until now there was a lack of experimental results using real image data to prove this conceptual solution. With this paper, the very first experimental work is presented on the basis of a new ray tracing model approach, which considers more accurate micro image centre positions. To evaluate the developed method, the blur metric of objects in a refocused image stack is measured and compared with proposed predictions. The results suggest quite an accurate approximation for distant objects and deviations for objects closer to the camera device.
\end{abstract}

Index Terms - Light field, plenoptic, camera, refocusing, distance estimate

\section{INTRODUCTION}

The plenoptic camera gained a lot of attention after its historical debut in the early years of the last century [1]. Since then, its conceptual setup relies on the basic principle of placing a Micro Lens Array (MLA) in front of an image acquisition device. The major contributions that let revive the plenoptic camera technology were the integration of a digital sensor [2], the four-dimensional (4-D) light field parameterisation [3], the discovery of digital refocusing [4] and the first successful implementation of refocusing using a plenoptic camera [5]. Subsequent research has been carried out in many directions including the investigation of different MLA focus settings [6]. In this paper the focus is turned on the Standard Plenoptic Camera (SPC) setup in which the image sensor is placed at the distance of the micro lens focal length. A well known example of such a camera type is the light field camera from Lytro initially launched in 2012. Yet, Lytro's cameras do not supply the feature of estimating the distance at which the user is refocusing. In our previous work [7], we developed a promising method to trace rays through the SPC and to address the problem about the refocusing distance of a digitally focused photograph. Nonetheless, this work has suffered from rather weak experimental validation. This came as a result of an imprecise micro image centre approximation used in the proposed ray tracing model.

The work presented in this paper builds on the previous development [7] by refining the ray tracing model and providing the first distance estimation results based on real captured data. In order to validate our proposed method, we first compute a stack of refocused images, then measure the blur metric of objects at a known distance and compare them with the estimated focusing distance to see whether the blur metric results match our predictions.

\section{STANDARD PLENOPTIC CAMERA MODEL}

Previously, the authors have shown in [7] how to trace light rays involved to synthesise a refocused image. One of the assumptions made in this model is that each micro image centre lies on the optical axis of its respective micro lens. The detection of micro image centres is crucial as they serve as reference origins in the image synthesis process. Related work carried out by Dansereau et al. [8] suggested that the central micro image location deviates from the micro lens optical axis as depicted in Fig. 1. This is due to the finite separation distance between main lens and MLA resulting in a shift, denoted as $e$, between micro lens centres and the respective micro lens axis.

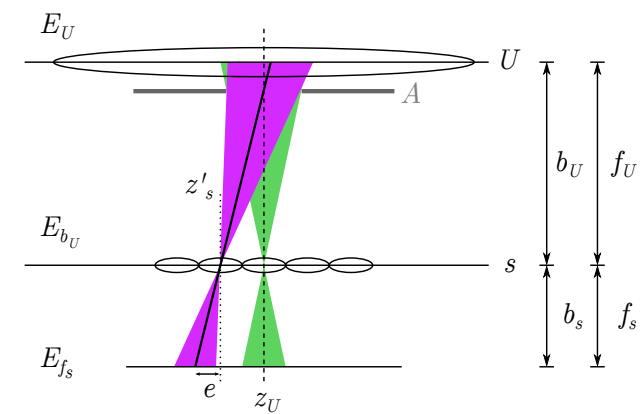

Figure 1: Main lens aperture calibration. Note that image centres deviate by $e$ from the optical axis of a micro lens.

Results presented in [7] do not reflect the error caused by a projective centre shift $e$. For that reason, we propose a refined model based on [7] while taking $e$ into account. In contrast to the previous model [7], chief rays at the micro image centres originated from the main lens optical centre as seen in Fig. 2. The micro image centres will be taken as a starting point for the orientation in the 4-D light field image.

According to Levoy and Hanrahan [3], a light field may be adequately parameterised by four variables $u, v, s, t$. Thereby, light field coordinate pairs $(s, t)$ and $(u, v)$ represent ray interesections at two two-dimensional (2-D) planes placed behind one another so that each ray passes through both. Similar to the statements made in [7], SPC refocusing can be efficiently accomplished in spatial domain by integrating pixels whose corresponding rays intersect in object space. 
If the raw plenoptic photograph $E_{f_{s}}$ is seen to contain the 4-D light field information indexed by $s=\{s, t\}$ for the 2-D spatial and $\boldsymbol{u}=\{u, v\}$ for the 2-D directional dimension, a synthesised refocused photograph $E_{a}^{\prime}$ is mathematically obtained by

$$
E_{a}^{\prime}\left[\boldsymbol{s}_{j}\right]=\sum_{i=-c}^{c} E_{f_{s}}\left[\boldsymbol{s}_{j+a(c-i)}, \boldsymbol{u}_{c+i}\right], \quad a \in \mathbb{R}_{\geq 0}
$$

where $j$ and $i$ are indices for micro lens $\boldsymbol{s}$ and micro image $\boldsymbol{u}$ samples, respectively. Let $\hat{m}$ denote the micro image resolution which is assumed to be homogeneous, its sample index $i$ counts within the range of $[-(\hat{m}-1) / 2,(\hat{m}-1) / 2]$. Thereby, $c=(\hat{m}-1) / 2$ denotes the centre of the micro image in case its resolution is seen to be homogeneous and odd. The synthesis Eq. (1) has been derived from a combination of positions $\left(\boldsymbol{s}_{j}, \boldsymbol{u}_{c+i}\right)$ in Fig. 2 that belong to rays intersecting in object space at a certain plane $a$. Depending on the object scene, light has potentially emitted from plane $a$ and distributed over micro images $\boldsymbol{s}_{j}$. To retrieve the intensity of light rays emanated from $a$, intensities $\boldsymbol{u}_{c+i}$ are integrated in Eq. (1). Note that this refocusing synthesis omits a commonly used intermediate step of generating viewpoint images and thus it takes less computation time.

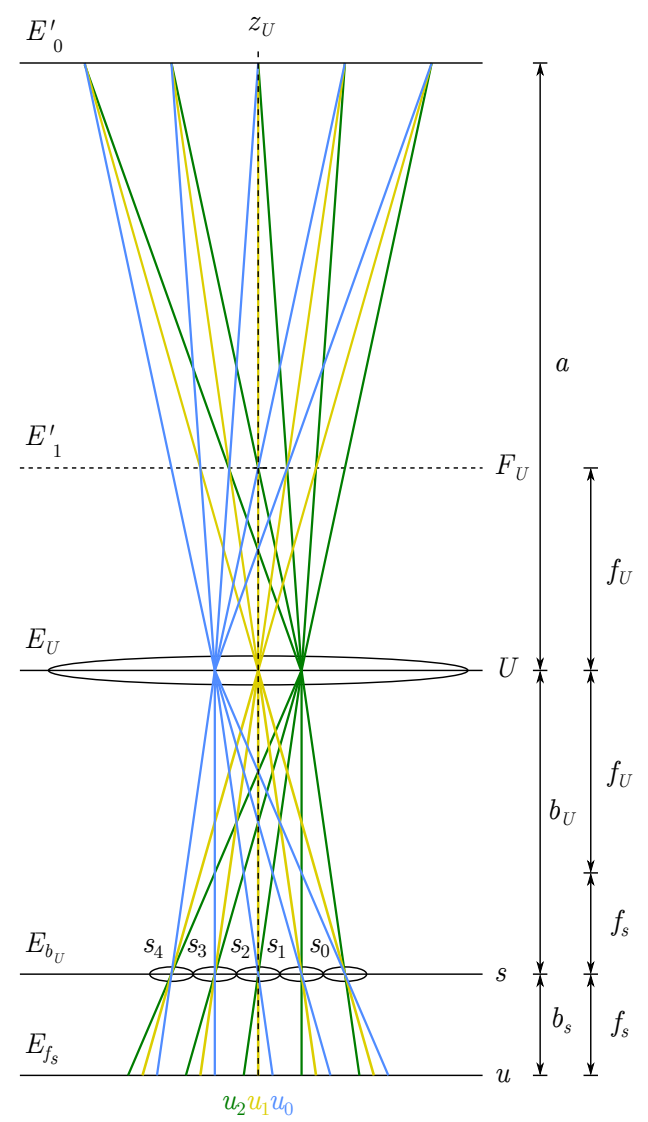

Figure 2: Standard plenoptic camera ray tracing model. The main lens $U$ is depicted as a thin lens. For clearness, ray tracing is performed with chief rays only.

\section{RAY TRACING EQUATIONS}

As the optical centre of the respective micro lens does not represent the micro image centre, we propose here a novel model that will take into account this displacement. More detailed explanations on the derivation of the previous model can be found in [7].
To start tracing light rays through the camera, an appropriate pair of ray positions intersecting at a desired plane $a$ needs to be selected in Fig. 2. Once the ray pair is chosen, its corresponding ray positions on the imaging device need to be estimated. The following descriptions apply to both intersecting rays equally. First, the optical center of the parent micro lens of a ray is given by

$$
s_{j}=j \times p_{\hat{m}}
$$

where the index $j$ is seen to start to count from the micro lens closest to the main lens optical axis. Since rays impinging on the micro image centre intersect the optical centres of the main lens and micro lens, their slope $m_{c, j}$ may be obtained by

$$
m_{c, j}=-\frac{s_{j}}{b_{U}}
$$

in which the image distance $b_{U}$ separates $U$ and $s$ from one another. To find a micro image centre $u_{c, j}$ on the sensor plane, the respective central ray slope $m_{c, j}$ will be extended in a way that it intersects the sensor by calculating

$$
u_{c, j}=-m_{c, j} \times f_{s}+s_{j}
$$

where $f_{s}$ denotes the micro lens focal length. Provided that the adjacency relation $i$ and the pixel pitch $p_{p}$ are known, the selected ray position $u_{i, j}$ on the sensor is found by

$$
u_{i, j}=u_{c, j}+i \times p_{p}
$$

Given the ray sensor position $u_{i, j}$, its corresponding slope is

$$
m_{i, j}=\frac{s_{j}-u_{i, j}}{f_{s}}
$$

By definition, chief rays travel through the optical centres of lenses, so that the next plane at which rays change their slope is $U$. The intersection with $U$ can be written as

$$
U_{i, j}=m_{i, j} \times b_{U}+s_{j}
$$

Due to the focus setting of the MLA, each ray underneath a micro image can be seen to result in a beam of parallel light rays in the range between $s$ and $U$. Thus, these parallel light rays originated from a point along the focal plane of the main lens $U$. Knowing the main lens focal length $f_{U}$, the object space position at which rays converge may be obtained by

$$
F_{i, j}=m_{i, j} \times f_{U}
$$

Hence, the ray slope $q_{i, j}$ in object space follows from the intersections at $U$ and $F_{U}$ such that

$$
q_{i, j}=\frac{F_{i, j}-U_{i, j}}{f_{U}}
$$

The path of a ray may be described by a linear function of the depth $z$ which would be of the form

$$
\widehat{f}_{i, j}(z)=q_{i, j} \times z+U_{i, j}, \quad z \in[U, \infty)
$$

Having the functions $\widehat{f}$ of two rays $g$ and $h$ that converge in a plane $a$, their intersection is given by solving the equation of both

$$
\widehat{f}_{g}(z)=\widehat{f}_{h}(z), \quad z \in[U, \infty)
$$

Letting the solution be $z_{U}$, its numeric value merely represents the distance from plane $U$ to its corresponding intersection at refocusing plane $a$. To compute the overall distance $d_{a}$ from the image plane of the sensor to plane $a$, respective optical parameters must therefore be added. This may be achieved by

$$
d_{a}=f_{s}+\overline{H_{1 s} H_{2 s}}+b_{U}+\overline{H_{1 U} H_{2 U}}+z_{a}
$$

where $\overline{H_{1 s} H_{2 s}}$ and $\overline{H_{1 U} H_{2 U}}$ represent the separation between principal planes of micro lens and main lens, respectively. 


\section{EXPERIMENTAL WORK}

In this section it will be shown how experiments are conducted to obtain quantitative results supporting the claimed solution. The major problem in using commercially available plenoptic cameras to prove our suggested concept is that some parameters, e.g. the principal plane locations $\overline{H_{1 U} H_{2 U}}$ or the image distance $b_{U}$, are unknown as manufacturers do not disclose the entire camera specification. To overcome that issue, our team designed a custom plenoptic camera. All camera parameters necessary for the computation of the equations in Section 3 can be found in Table 1 .

Table 1: Standard plenoptic camera parameters [mm]

\begin{tabular}{cccccc}
\hline$p_{p}$ & $p_{\hat{m}}$ & $f_{s}$ & $\overline{H_{1 s} H_{2 s}}$ & $f_{U}\left(=b_{U}\right)$ & $\overline{H_{1 U} H_{2 U}}$ \\
\hline 0.009 & 0.125 & 2.75 & 0.396 & 193.294 & -65.556 \\
\hline
\end{tabular}

For the experimental setup, the main lens $U$ is focused to infinity such that $b_{U}=f_{U}$. Besides, markers have been placed in the scene labelled with numbers indicating the distance to the camera device. However, note that, in our experimental setup, the image sensor is positioned $43.646 \mathrm{~mm}$ away from the distance measurement origin and, thus, the indicated numbers for each marker are increased by this shift to reflect the real distance between the markers and the image sensor. For instance, a marker labelled with 300 is separated by $304.3646 \mathrm{~cm}$ from the image sensor.

Table 2 provides the calculated metric distance $d_{a}$ at a given refocusing slice $a$ of the proposed and previous [7] attempt. By comparing the results of both methods, it may be obvious that the deviation gets significantly greater when approaching the camera device. To validate the accuracy of predictions, real images are used. Refocused images are processed according to Eq. (1). An example of a refocused photograph from our test series is given in Fig. 3. Note that the refocusing process implies an interpolation of whole micro images in case $a$ is a positive real number. By expressing the real number as a fraction, the denominator represents

Table 2: Predicted $d_{a}[\mathrm{~cm}]$

the upscaling factor. In our test measurement, a linear interpolation is chosen for the vertical as well as the horizontal dimension.

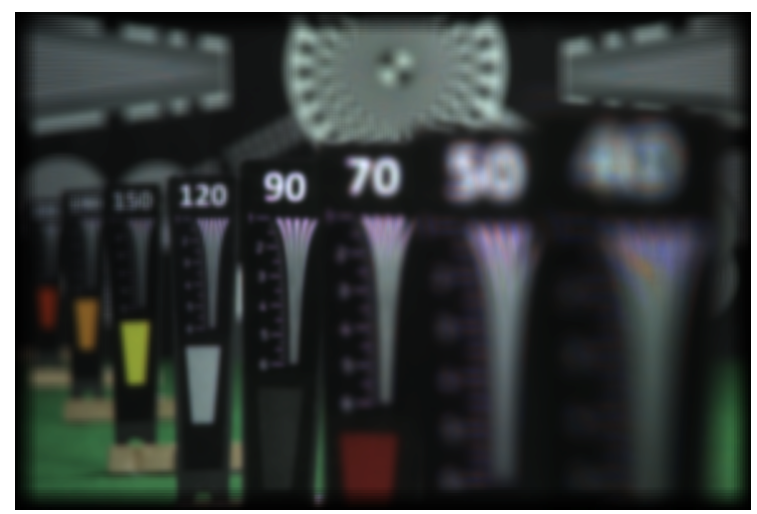

Figure 3: Exemplary refocused photograph. The image has been computed via Eq. (1) using refocusing slice $a=10 / 9$.

\begin{tabular}{|c|c|c|c|}
\hline$a$ & $\begin{array}{l}\text { proposed } \\
d_{a}\end{array}$ & $\begin{array}{c}\text { previous } \\
d_{a}[7]\end{array}$ & $\begin{array}{l}\text { The evaluation is accomplished by extracting the region of in- } \\
\text { terest (ROI) for a particular object in each image of the refocused }\end{array}$ \\
\hline $0 / 9$ & Inf & Inf & stack. Figure 4 depicts a ROI at $304.3646 \mathrm{~cm}$ distance expected to \\
\hline $1 / 9$ & 897.9 & 917.2 & exhibit best focus at slice $a=3 / 9$. Closer inspection reveals how \\
\hline $2 / 9$ & 457.7 & 477.0 & the degree of blur changes when $a$ varies in Eq. (1). The ROIs \\
\hline $3 / 9$ & 310.9 & 330.2 & are cropped in a way that the bounding boxes for each respective \\
\hline $4 / 9$ & 237.6 & 256.9 & region are aligned to ensure correct refocusing measurements. \\
\hline
\end{tabular}

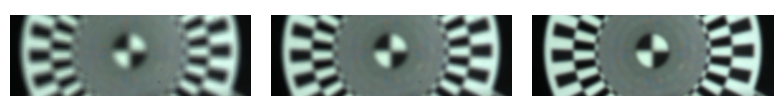
(a) $a=0 / 9$,
$S=0.64$
(b) $a=1 / 9$,
$S=0.80$
(c) $a=2 / 9$, $S=0.93$

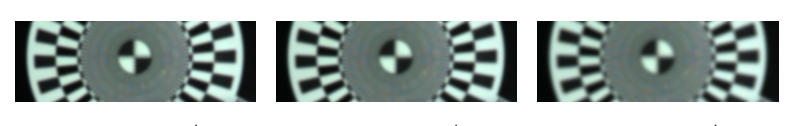
(d) $a=3 / 9$,
$S=1.0$
(e) $a=4 / 9$,
$S=0.96$
(f) $a=5 / 9$
$S=0.84$

\begin{tabular}{l|l|l|c}
$15 / 9$ & 76.1 & 95.5 & $S=1.0$ \\
\hline $16 / 9$ & 72.5 & 91.8 \\
\hline $17 / 9$ & 69.2 & 88.6 \\
\hline $18 / 9$ & 66.4 & 85.7 \\
\hline $19 / 9$ & 63.8 & 83.1 \\
\hline
\end{tabular}

\begin{abstract}
(g) $a=6 / 9$,
$S=0.68$
\end{abstract}
(h) $a=7 / 9$,
$S=0.53$

$S=0.39$

Figure 4: Region of refocused photographs. The object plane is placed at $304.3646 \mathrm{~cm}$ distance from the image sensor. $S$ denotes the measured sharpness. The denominator in $a$ indicates the upsampling factor for the linear interpolation of whole micro images.

To quantitatively assess the degree of focus in the previously extracted regions of refocused photographs, we deploy a slight modification of the method proposed by Mavridaki and Mezaris [9]. We transform the ROI using the 2-D Discrete Fourier Transformation where $k$ and $l$ are pixels ranging from $0, \ldots, K-1$ and $0, \ldots, L-1$, respectively. To obtain the magnitude, we compute

$$
\mathcal{X}[n, m]=\left|\sum_{k=0}^{K-1} \sum_{l=0}^{L-1} x[k, l] e^{-2 \pi i(k n / K+l m / L)}\right|
$$




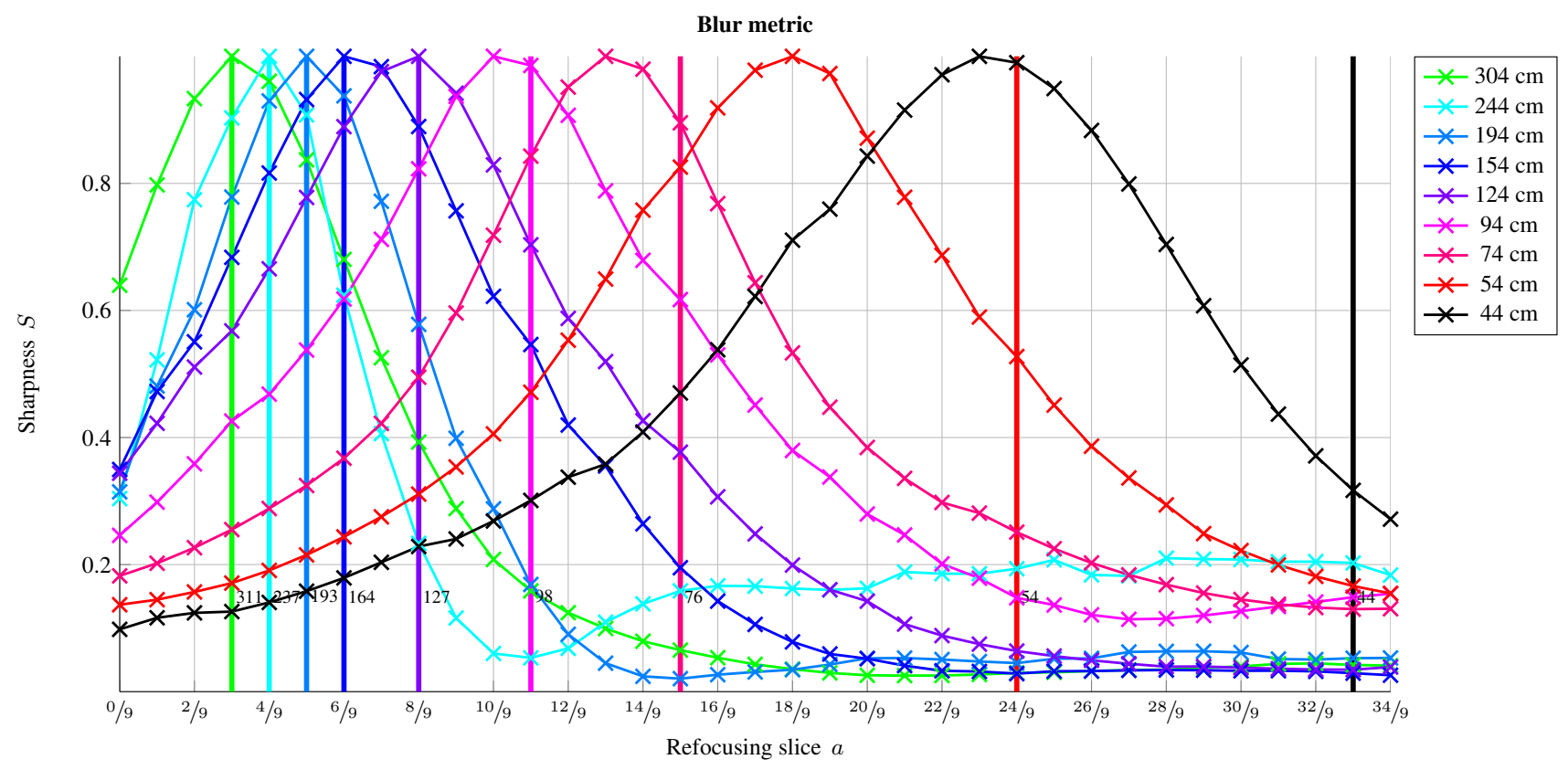

Figure 5: Blur metric of refocused photographs. The main lens focuses to infinity $\left(b_{U}=f_{U}\right)$. Vertical lines indicate the expected position of best measured focus for respective markers. All marker distances are given in centimeters, although note that the image sensor is placed $4.3646 \mathrm{~cm}$ away from the measurement origin and therefore marker lines take this shift into account.

In the next step, the overall power $T E$ of the magnitude is obtained by

$$
T E=\sum_{n=0}^{N-1} \sum_{m=0}^{M-1} \mathcal{X}[n, m]^{2}
$$

In order to measure the power $E$ of high frequency parts of the magnitude, we calculate

$$
E=T E-\sum_{n=0}^{4} \sum_{m=0}^{4} \mathcal{X}[n, m]^{2}
$$

Lastly, the ratio of the magnitude powers yield the sharpness $S$

$$
S=\frac{E}{T E}
$$

Results are normalised and plotted in Fig. 5. As seen in the diagram, the proposed prediction provides a fair estimation for objects at a far distance. However, deviations occur at close objects and are believed to be due to optical distortions and aberrations. These are subject to further investigation. Recalling Table 2, it may be apparent that the proposed solution performs better in estimating the refocusing distance than [7].

\section{CONCLUSION}

To the best of our knowledge, the work carried out in this paper has provided the first experimental results in verifying the predicted distance of refocused objects. It has been shown that the refocusing distance estimation matches the measured blur metric for far objects, although deviations exist for objects near to the camera. Nevertheless, the suggested approach performs significantly better than the previous attempt. Further research may provide a ground truth reference distance from a real ray simulation tool. Besides, it is worth to investigate different main lens focus settings as well as depth of field boundaries in the refocused photographs.

\section{REFERENCES}

[1] G. Lippmann, "Épreuves réversibles donnant la sensation du relief," Académie Des Sciences, pp. 446-451, March 1908.

[2] E. H. Adelson and J. Y. Wang, "Single lens stereo with a plenoptic camera," IEEE Transactions on Pattern Analysis and Machine Intelligence, vol. 14, no. 2, pp. 99-106, February 1992.

[3] M. Levoy and P. Hanrahan, "Light field rendering," Tech. Rep., Stanford University, 1996.

[4] A. Isaksen, L. McMillan, and S. J. Gortler, "Dynamically reparameterized light fields," in Proceedings of the 27th Annual Conference on Computer Graphics and Interactive Techniques, New York, NY, USA, 2000, SIGGRAPH '00, pp. 297-306, ACM Press/Addison-Wesley Publishing Co.

[5] R. Ng, M. Levoy, M. Brèdif, G. Duval, M. Horowitz, and P. Hanrahan, "Light field photography with a hand-held plenoptic camera," Tech. Rep. CTSR 2005-02, Stanford University, 2005.

[6] A. Lumsdaine and T. Georgiev, "Full resolution lightfield rendering," Tech. Rep., Adobe Systems, Inc., January 2008.

[7] C. Hahne, A. Aggoun, S. Haxha, V. Velisavljevic, and J. C. J. Fernández, "Light field geometry of a standard plenoptic camera," Opt. Express, vol. 22, no. 22, pp. 26659-26673, Nov 2014.

[8] D. G. Dansereau, O. Pizarro, and S. B. Williams, "Decoding, calibration and rectification for lenselet-based plenoptic cameras," in 2013 IEEE International Conference on Computer Vision and Pattern Recognition (CVPR), June 2013, pp. 1027 - 1034 .

[9] E. Mavridaki and V. Mezaris, "No-reference blur assessment in natural images using fourier transform and spatial pyramids," in 2014 IEEE International Conference on Image Processing (ICIP), Oct 2014, pp. 566-570. 